\title{
EVALUATION OF THE OBESITY INFLUENCE ON THE LIFE QUALITY IN PATIENTS WITH BRONCHIAL ASTHMA
}

\author{
Yulia Zaikina \\ Department of Phthisiology, Pulmonology and Family Medicine ${ }^{l}$ \\ julia.ua.h@gmail.com \\ Nataliia Cherednichenko \\ Department of Internal Medicine with the Center for Respiratory Medicine \\ Sumy State University \\ 2 Rymskoho-Korsakova, Sumy, Ukraine, 40007 \\ natalya.cheredn@gmail.com \\ Hanna Tymchenko \\ Department of Phthisiology, Pulmonology and Family Medicine ${ }^{l}$ \\ hanna.zaprovalna@gmail.com \\ Gennadii Kochuiev \\ Department of General Practice - Family Medicine ${ }^{1}$ \\ docentik1961@gmail.com \\ Maryna Kochuieva \\ Department of Internal Medicine \\ V. N. Karazin Kharkiv National University \\ 4 Svobody sq., Kharkiv, Ukraine, 61022 \\ kochuevamarina@gmail.com \\ ${ }^{1}$ Kharkiv Medical Academy of Postgraduate Education \\ 58 Amosova str., Kharkiv, Ukraine, 61176
}

Abstract

The obese asthma is characterized by a more severe course. The feature of this comorbid condition is the reduced effectiveness of basic therapy with inhaled corticosteroids, which prevents optimal control of symptoms, requires increasing doses and increases the number of exacerbations and hospitalizations. The problem of life quality (LQ) research in patients with asthma and obesity is highly relevant today, which made it the reason for this study.

The aim of the study was to determine the effect of obesity on LQ in patients with asthma.

Materials and methods. We selected for participating in the study 46 patients with moderate severity asthma. Examination of the patients included: objective examination with an anthropometric evaluation, the test of the LQ with two questionnaires: general one - Medical Outcome Study SF-36 (MOS SF-36) and specialized Asthma Quality of Life Questionnaire (AQLQ).

Results. The increase in body mass index (BMI) in patients with asthma according to the questionnaires MOS SF-36 and AQLQ is associated with significant reductions in viability $(r=-0.33, p<0.05)$, physical activity $(r=-0.37, p<0.01)$, social activity $(r=-0.36, p<0.01)$ and the degree of reduction of the patient's tolerance to adverse environmental factors $(r=-0.29, p<0.05)$.

Conclusions. The presence of concomitant obesity in patients with asthma is associated with significant reductions in the parameters of physical and social activity, viability and with an increase in the subjective pain assessment (according to the questionnaire MOS SF-36) and characterized by significantly lower rates of activity, tolerance to adverse environmental factors and general life quality (according to the AQLQ questionnaire).

Keywords: asthma, obesity, life quality, AQLQ questionnaires, MOS SF-36 questionnaire, BMI.

DOI: $10.21303 / 2504-5679.2021 .001842$

\section{Introduction}

Chronic respiratory diseases consider being one of the leading in terms of prevalence, disability, and mortality in the world. Asthma occupies an important place among them, and remains one of the most difficult medical and social problems nowadays. According to the WHO, 
the number of patients with asthma in the world is over 330 million people and continues to grow steadily. The disease affects all age groups $[1,2]$.

In turn, the number of asthma patients in combination with obesity is growing, which is associated with an increase in the number of overweight people. According to various authors, between 36.7 and $44 \%$ of asthma patients are obese [2, 3]. The results of epidemiological studies show that the presence of obesity almost 2 times increases the chances of asthma developing and, if previously this information was contradictory, now the accumulated data have confirmed this hypothesis [4]. The physiology of respiration is changed because of the excessive development of the adipose tissue: it leads to mechanical compression of the diaphragm, reduced lung volume, reduces the flexibility and strength of the respiratory muscles, and increases lung resistance [5, 6]. Obesity leads to systemic inflammation [7]. Adipose tissue is metabolically active and produces many hormone-like substances, cytokines, chemokines, which act locally and systemically, and also produces a large number of inflammatory mediators, which suggests an immunological link between obesity and asthma [8, 9]. An important role belongs to leptin, experimental studies proved its provision the pathogenetic mechanism of the relationship between obesity and asthma and supports the inflammatory process in the bronchi [10].

The obese asthma is characterized by a more severe course. The feature of this comorbid condition is the reduced effectiveness of basic therapy with inhaled corticosteroids, which prevents optimal control of symptoms, requires increasing doses and increases the number of exacerbations and hospitalizations $[9,11]$. In this regard, GINA experts have identified a separate asthma with obesity phenotype $[1,11,12]$. Like any other comorbidity, the combination of asthma with obesity entails significant emotional, physical, and financial strains leading to the deterioration in the patient's life quality (LQ). If earlier there was a tendency to focus exclusively on the symptoms of the disease, now the WHO recommends taking into account the priorities of modern medicine and aim not only to eliminate symptoms and normalize clinical and functional parameters, but to consider the improvement of the life quality as the main criterion [9, 11]. LQ is an integral indicator that reflects the degree of a person's adaptation to the disease and the ability to perform the usual functions corresponding to his social status. The study of LQ allows evaluation of the physical, social, and psychological functioning of the patient, also giving an idea of how the disease influences his condition. It is known from the literature that patients with a combination of obesity and bronchial asthma have worse LQ rates than those with normal weight, and the number of the examined people with LQ below average is twice as high among overweight patients [13, 14]. The problem of LQ research in patients with bronchial asthma and obesity is highly relevant today, which made it the reason for this study.

The aim of the study - to determine the effect of obesity on LQ in patients with asthma.

\section{Materials and methods}

The research was conducted on the basis of the pulmonology department of the Municipal Non-commercial Enterprise «City Clinical Hospital No. 13» of Kharkiv City Council (Kharkiv, Ukraine) during 2018-2020. We examined 46 patients with moderate severity asthma, all women aged 35-50, and the average age was $45.2 \pm 0.89$ years. Diagnosis of asthma, the allocation of its severity was performed according to GINA recommendations [7].

The study was held according to the Helsinki Declaration adopted by the General Assembly of the World Medical Association (1964-2000), The Convention for the European Union on Human Rights and Biomedicine, relevant provisions of the WHO, and laws of Ukraine, and was approved by a local ethics committee of Kharkov Medical Academy of Postgraduate Education (protocol No. 1, from 11 Feb., 2021). All patients signed informed consent for participating in the clinical trial.

All patients underwent the general clinical examination, which included history taking and objective examination with an anthropometric evaluation of body mass $(\mathrm{kg})$, height $(\mathrm{m})$, waist circumference $(\mathrm{WC}, \mathrm{cm})$, hip circumference $(\mathrm{HC}, \mathrm{cm})$, followed by a body mass index calculation $\left(\mathrm{BMI}, \mathrm{WHO}, \mathrm{kg} / \mathrm{m}^{2}\right.$ ) and the relative circumference of the waist to the circumference of the hips $(\mathrm{WC} / \mathrm{HC})$. According to the body mass index $\left(\mathrm{BMI}=\right.$ body weight, $\left.\mathrm{kg} / \mathrm{height}, \mathrm{m}^{2}\right)$ all patients were divided into two groups. The first group consisted of patients with BMI from 18.5 to 
$24.9 \mathrm{~kg} / \mathrm{m}^{2}$ that is the normal weight and the second group was the patients with obesity (BMI from $30.0 \mathrm{~kg} / \mathrm{m}^{2}$ and more).

All patients were treated with standard basic therapy of inhalation corticosteroids (IGCS) and $\beta_{2}$-agonists of the prolonged action, according to the degree of illness severity. The groups were comparable in age and asthma severity.

Exclusion criteria were: diabetes mellitus, chronic infectious, systemic, oncological and psychiatric diseases, and chronic heart failure with a left ventricular ejection fraction of less than $55 \%$, the presence of a history of myocardial infarction, stroke and signs of ischemic heart disease, heart valve regurgitation more than 2 degree.

To test the LQ of patients with asthma with obesity we used two questionnaires about life quality: general one - Medical Outcome Study SF-36 (MOS SF-36) (Ware J. E., 1992) and specialized Asthma Quality of Life Questionnaire (AQLQ) (Juniper E. F., 1995). With the questionnaire MOS SF-36 an assessment of LQ was carried out in points on the scales: physical activity, the role of physical problems in limiting vital functions, pain, general health, viability, social activity, the role of emotional problems in limiting vital functions, mental health. On the basis of the results of the AQLQ questionnaire 5 indicators of the LQ were shown: limitation of activity (activity), the severity of the main symptoms of asthma (symptoms), the degree of asthma influence on the patient's psycho-emotional sphere (emotions), the degree of patient's tolerance to adverse environmental factors (environment), general life quality (GLQ). The survey was carried out by the questionnaire method based on the direct experience of the respondents.

Parametric and non-parametric methods were used to compare the central parameters of the groups: Student's t-test, Wilcoxon tests (W) and Mann-Whitney (MU). The pairwise comparison of groups used the criterion U - Mann-Whitney (MU), to compare the occurrence of a feature in the frequency analysis in groups used the Pearson test $\chi$-square $\left(\chi^{2}\right)$. For all statistical values, levels of importance were taken into account $(p) 0.05 ; 0.01 ; 0.001$.

\section{Results}

Analyzing the data of LQ when using the questionnaire SF-36 in the group of obese patients, a significant decrease in the parameters of physical and social activity was set, also noted an increase in respondent's subjective assessment of pain in limiting life compared to patients with normal body weight (Table 1). It is known that pain is a non-typical symptom of asthma. Even minor, but long-lasting physical discomfort in the form of breath shortness can be perceived by patients as pain. Probably, this is due to the lack of mechanisms of adaptation to this kind of feeling [7].

Table 1

Life quality indicators according to the SF-36 questionnaire $(M \pm m)$

\begin{tabular}{ccc}
\hline Indicators (in points) & Group 1 $\boldsymbol{n}=\mathbf{2 4}$ & Group 2 $\boldsymbol{n}=\mathbf{2 2}$ \\
\hline Physical activity & $73.1 \pm 2.4$ & $59.6 \pm 3.2^{*}$ \\
The role of physical problems in limiting vital functions & $53.8 \pm .4 .6$ & $45.4 \pm 5.5$ \\
General health & $75.0 \pm 3.2$ & $61.3 \pm 3.6^{*}$ \\
Viability & $47.1 \pm 2.3$ & $45.4 \pm 2.2$ \\
Social activity & $60.5 \pm 2.4$ & $51.6 \pm 3.1^{*}$ \\
The role of emotional problems in limiting life & $68.4 \pm 2.8$ & $60.7 \pm 2.9$ \\
Mental health & $63.2 \pm 4.4$ & $56.3 \pm 4.7$
\end{tabular}

Note: * - reliability of differences in indicators compared with a group of patients with normal body weight

Comparison of the parameters of the general LQ in patients with normal body weight and obesity revealed a significant deterioration in viability, as well as a significant decrease in selfesteem in terms of physical and social activity. 
Analysis of AQLQ questionnaire and comparison of patient's self-esteem clearly showed the impact of obesity on such areas as «general activity» and «environment»; these indicators were significantly higher in patients with normal body weight (Table 2). In the group of obese patients, the lowest was the activity rate, which scale varied from «moderate» to «absolute», there was also a significant decrease in the parameter «environment».

Table 2

Life quality indicators according to the AQLQ questionnaire $(M \pm m)$

\begin{tabular}{ccc}
\hline Indicators (in points) & Group $\mathbf{1} \boldsymbol{n}=\mathbf{2 4}$ & Group 2 $\boldsymbol{n = 2 2}$ \\
\hline Activity & $4.1 \pm 0.2$ & $3.6 \pm 0.2^{*}$ \\
Symptoms & $4.2 \pm 0.2$ & $4.1 \pm 0.3$ \\
Emotions & $4.5 \pm 0.3$ & $3.8 \pm 0.1$ \\
Environment & $4.8 \pm 0.1$ & $3.7 \pm 0.3^{*}$ \\
General LQ & $4.2 \pm 0.3$ & $3.9 \pm 0.1^{*}$
\end{tabular}

Note: * - reliability of differences in indicators compared with a group of patients with normal body weight

A correlation analysis in the group of obese patients, questionnaire SF-36, found a significant negative effect of BMI on the indicators of «viability» $(r=-0.33, p<0.05)$, "physical activity» $(r=-0.37, p<0.01)$, «social activity» $(r=-0.36, p<0.01)$, as well as the impact on subjective health assessment by the parameter «environment» $(r=-0.29, p<0.05)$. The obtained results clearly demonstrated the negative impact of obesity on LQ and the state of the psycho-emotional status in patients with asthma. In obese patients significantly reduced physical and psychosocial components of health self-esteem.

\section{Discussion}

The study assessed the main aspects of LQ in asthma with obesity patients, and demonstrated the influence of obesity on the general LQ and specific health. It was noted that LQ in asthma with obesity patients was significantly lower than in subjects with normal body weight, both in terms of physical functioning and social life of patients. It is consistent with previous studies [13, 16-17] and confirms the assumption of the obesity influence on both physical and psychosocial aspects of life quality in patients with asthma.

In obese patients the physical and psychosocial components of health self-esteem were significantly reduced. Among the parameters relevant to physical health, the lowest were the parameters of physical activity and pain perception. Some authors suggest that the subjective sensation of pain can be explained by the influence of prolonged stress, which can lead to the appearance of breath shortness feeling without objective signs of increased bronchial obstruction $[12,16]$.

Obesity significantly reduces the level of general and specific LQ of people with asthma, especially in the spheres of physical and social functions [18, 19]. All the above-mentioned allow considering obesity in asthma patients to be one of the main reasons for the LQ worsening [20]. The results of the study showed that the BMI value helps to get objective information about the patient's health. Simplicity and accessibility of the LQ parameters value and the assessment of the health with the additional questionnaire of the asthma patients on the basis of obesity allow to widespread use of the data in the medical practice.

The limitations of the research. The present study was intended to investigate only the quality of life of asthma patients with concomitant obesity and did not include analysis of clinical and laboratory parameters for these patient groups, as well as the study of patients with other chronic respiratory diseases and variants of comorbidity.

The further research plan to study the influence of program of complex treatment on LQ of obese asthma patients. 


\section{Conclusions}

1. The method of questioning patients with asthma and asthma with obesity patients using questionnaires MOS SF-36 and AQLQ allows to study in detail the features of LQ reduction and to determine the contribution of each pathology separately.

2. The presence of concomitant obesity in patients with asthma when surveyed by the questionnaire MOS SF-36 is associated with significant reductions in the parameters of physical and social activity, viability and with an increase in the subjective pain assessment.

3. Asthma with obesity patients according to the AQLQ questionnaire in comparison with patients with asthma with normal body weight are characterized by significantly lower rates of activity, tolerance to adverse environmental factors and general life quality.

4. The increase in BMI in patients with asthma according to the questionnaires MOS SF-36 and AQLQ is associated with significant reductions in viability $(r=-0.33, p<0.05)$, physical activity $(r=-0.37, p<0.01)$, social activity $(r=-0.36, p<0.01)$ and the degree of reduction of the patient's tolerance to adverse environmental factors $(r=-0.29, p<0.05)$.

\section{Conflicts of interest}

The authors declare there is no conflict of interest.

\section{Acknowledgments}

The scientific work is a fragment of research work of the Department of Tuberculosis, Pulmonology and Family Medicine of Kharkiv Medical Academy of Postgraduate Education «Cell-molecular and neurohumoral mechanisms of remodelling of target organs, their relationships and correction in patients with combined pathology», state registration number 0117 U006894.

\section{References}

[1] 2020 GINA main report - Global initiative for asthma (2020). Available at: https:/ginasthma.org/gina-reports/ Last accessed: 24.04.2021

[2] Bantulà, M., Roca-Ferrer, J., Arismendi, E., Picado, C. (2021). Asthma and Obesity: Two Diseases on the Rise and Bridged by Inflammation. Journal of Clinical Medicine, 10 (2), 169. doi: http://doi.org/10.3390/jcm10020169

[3] Denlinger, L. C., Phillips, B. R., Ramratnam, S., Ross, K., Bhakta, N. R., Cardet, J. C. et. al. (2017). Inflammatory and Comorbid Features of Patients with Severe Asthma and Frequent Exacerbations. American Journal of Respiratory and Critical Care Medicine, 195 (3), 302-313. doi: http://doi.org/10.1164/rccm.201602-0419oc

[4] Poulain, M. (2006). The effect of obesity on chronic respiratory diseases: pathophysiology and therapeutic strategies. Canadian Medical Association Journal, 174 (9), 1293-1299. doi: http://doi.org/10.1503/cmaj.051299

[5] Yashina, L. A. (2011). Overweight, obesity and lung pathology: The view of a pulmonologist. Health of Ukraine, 2 (14), 14-15.

[6] Svenningsen, S., Nair, P. (2021). There is more to severe asthma associated with obesity than inflammation. Respirology, 26 (4), 288-289. doi: http://doi.org/10.1111/resp.14012

[7] Sukhan, V. S. (2018). Comorbidity of bronchial asthma and obesity. Ukraine. Nation's Health, 2 (49), 70-72.

[8] Giesler, A., Mukherjee, M., Radford, K., Janssen, L., Nair, P. (2018). Modulation of human airway smooth muscle biology by human adipocytes. Respiratory Research, 19 (1). doi: http://doi.org/10.1186/s12931-018-0741-Z

[9] Tashiro, H., Takahashi, K., Sadamatsu, H., Kurihara, Y., Haraguchi, T., Tajiri, R. et. al. (2020). Biomarkers for Overweight in Adult-Onset Asthma. Journal of Asthma and Allergy, 13, 409-414. doi: http://doi.org/10.2147/jaa.s276371

[10] Ulrik, C. S. (2015). Asthma symptoms in obese adults: The challenge of achieving asthma control. Expert Review of Clinical Pharmacology, 9 (1), 5-8. doi: http://doi.org/10.1586/17512433.2016.1093415

[11] Ignatova, H. L., Makarova, E. A. (2016). Bronchial asthma and obesity: Clinical and pathogenetic aspects of the selection of a new phenotype of the disease. Modern Problems of Science and Educatio, 4, 16-19.

[12] Yashina, L. A., Ishchuk, S. G. (2011). Bronchial asthma in obese patients, features of the disease phenotype. Asthma and Allergy, 4, 46-49.

[13] Pertseva, T. A., Nudga, N. P. (2011). Asthma and obesity: What is the relationship? Ukrainian Pulmonology Journal, $1,61-64$.

[14] Baffi, C. W., Wood, L., Winnica, D., Strollo, P. J., Gladwin, M. T., Que, L. G., Holguin, F. (2016). Metabolic Syndrome and the Lung. Chest, 149 (6), 1525-1534. doi: http://doi.org/10.1016/j.chest.2015.12.034 
[15] Mafort, T. T., Rufino, R., Costa, C. H., Lopes, A. J. (2016). Obesity: systemic and pulmonary complications, biochemical abnormalities, and impairment of lung function. Multidisciplinary Respiratory Medicine, 11 (1). doi: http://doi.org/10.1186/ s40248-016-0066-Z

[16] Singh, M., Gupta, N., Kumar, R. (2016). Effect of obesity and metabolic syndrome on severity, quality of life, sleep quality and inflammatory markers in patients of asthma in India. Pneumonologia i Alergologia Polska, 84 (5), $258-264$. doi: http://doi.org/10.5603/piap.2016.0032

[17] Khalid, F., Holguin, F. (2018). A review of obesity and asthma across the life span. Journal of Asthma, 55 (12), $1286-1300$. doi: http://doi.org/10.1080/02770903.2018.1424187

[18] Miethe, S., Karsonova, A., Karaulov, A., Renz, H. (2020). Obesity and asthma. Journal of Allergy and Clinical Immunology, 146 (4), 685-693. doi: http://doi.org/10.1016/j.jaci.2020.08.011

[19] Garcia-Rio, F., Alvarez-Puebla, M., Esteban-Gorgojo, I., Barranco, P., Olaguibel, J. (2019). Obesity and Asthma: Key Clinical Questions. Journal of Investigational Allergology and Clinical Immunology, 29 (4), 262-271. doi: http://doi.org/ 10.18176/jiaci.0316

[20] Wood, L. G. (2017). Diet, Obesity, and Asthma. Annals of the American Thoracic Society, 14 (Supplement_5), S332-S338. doi: http://doi.org/10.1513/annalsats.201702-124aw

Received date 26.04.2021

(C) The Author(s) 2021

Accepted date 17.05.2021

This is an open access article

Published date 25.05.2021

under the Creative Commons CC BY license

How to cite: Zaikina, Y., Cherednichenko, N., Tymchenko, H., Kochuiev, G., Kochuieva, M. (2021). Evaluation of the obesity influence on the life quality in patients with bronchial asthma. EUREKA: Health Sciences, 3, 31-36. doi: 10.21303/2504-5679.2021.001842 